\title{
Flow analysis of the low Reynolds number swimmer $C$. elegans
}

\author{
Thomas D. Montenegro-Johnson, ${ }^{1}$ David A. Gagnon, ${ }^{2}$ Paulo E. Arratia, ${ }^{2, *}$ and Eric Lauga ${ }^{1, \dagger}$ \\ ${ }^{1}$ Department of Applied Mathematics and Theoretical Physics, Centre for Mathematical Sciences, \\ University of Cambridge, Wilberforce Road, Cambridge CB3 OWA, UK \\ ${ }^{2}$ Department of Mechanical Engineering and Applied Mechanics, University of Pennsylvania, \\ Philadelphia, Pennsylvania 19104, USA
}

(Received 15 February 2016; published 9 September 2016)

\begin{abstract}
Swimming cells and microorganisms are a critical component of many biological processes. In order to better interpret experimental studies of low Reynolds number swimming, we combine experimental and numerical methods to perform an analysis of the flow field around the swimming nematode Caenorhabditis elegans. We first use image processing and particle tracking velocimetry to extract the body shape, kinematics, and flow fields around the nematode. We then construct a three-dimensional model using the experimental swimming kinematics and employ a boundary element method to simulate flow fields, obtaining very good quantitative agreement with experiment. We use this numerical model to show that calculation of flow shear rates using purely planar data results in significant underestimates of the true three-dimensional value. Applying symmetry arguments, validated against numerics, we demonstrate that the out-of-plane contribution can be accounted for via incompressibility and therefore simply calculated from particle tracking velocimetry. Our results show how fundamental fluid mechanics considerations may be used to improve the accuracy of measurements in biofluiddynamics.
\end{abstract}

DOI: 10.1103/PhysRevFluids.1.053202

\section{INTRODUCTION}

The study of microorganism and cell swimming has numerous applications in both industry and medicine, for instance, in the context of mammalian reproduction [1,2], biofuel production [3], and the design of artificial biomedical systems [4]. Many of these swimmers, such as mammalian spermatozoa, self-propel by generating traveling undulations along their body [5,6]. One such undulatory swimmer is the biological model organism Caenorhabditis elegans, a multicellular, free-living slender nematode worm found in soil environments.

The genetics and physiology of this nematode are well-studied. Its genome has been completely sequenced [7], and a complete cell lineage has been established [8]. The neuromuscular system of $C$. elegans controls its body undulations which allow it to swim, dig, and crawl through diverse environments. The wealth of available biological knowledge thus makes $C$. elegans an ideal candidate for investigations that combine aspects of biology, biomechanics, and the fluid mechanics of propulsion.

Recently $C$. elegans has been used extensively as a model system for experimental studies of propulsion, particularly at low Reynolds number, due to its simple planar swimming gait and size [9-15]. The nematode generates planar bending waves through contractions of its ventral and dorsal muscles, producing a quasi-two-dimensional (2D) traveling sine wave along its body [10,16,17]. At around $1 \mathrm{~mm}$ in length and $75 \mu \mathrm{m}$ in diameter, C. elegans is significantly larger than the majority of low-Re undulatory swimmers, enabling high-resolution reconstruction and analysis of planar flow fields from particle tracking data. The resulting flow fields can be used to probe properties of both the swimmer and fluid, providing new insights into the physics of undulatory propulsion $[11-15,18-20]$.

*parratia@seas.upenn.edu

†e.lauga@damtp.cam.ac.uk 


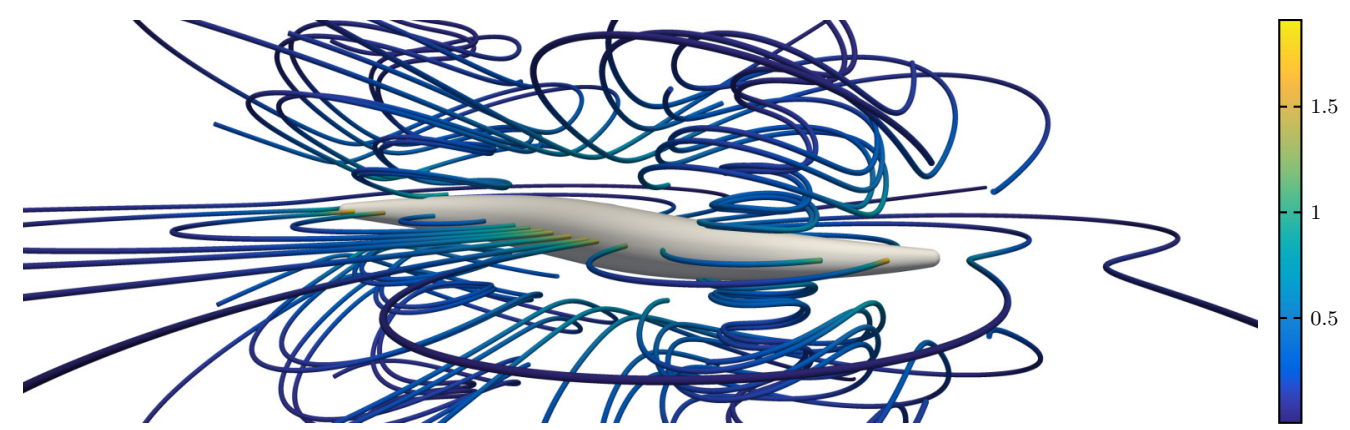

FIG. 1. Instantaneous flow streamlines generated by C. elegans, computed numerically from experimental waveform data. The streamlines show a complex, three-dimensional flow field. Color bar: speed mm/s.

However, despite exhibiting a planar swimming stroke, the flow around C. elegans has a complex three-dimensional structure (Fig. 1) which is difficult to capture experimentally. Many useful flow properties, such as the shear rate, are dependent upon out-of-plane flow contributions not measured by planar velocimetry data; in essence, one measures a 2D slice of a three-dimensional (3D) field. This limits, for example, our ability to accurately determine drag and propulsion forces experienced and exerted by the swimming nematode. In this work, we show that numerical and theoretical fluid mechanics techniques can be used to improve the processing and analysis of these experimental flow fields.

We first obtain detailed (experimental) imaging data on the shape and kinematics of a swimming C. elegans, together with particle-tracking data for flow field reconstruction. We then develop a numerical boundary element model of the nematode, with geometry and boundary conditions specified directly from the experiments, and use this model to improve the processing of particle tracking velocimetry results. Using simulated flow fields, we examine the spatial distribution of flow shear rate, an important quantity related to power dissipation and relevant for studies on locomotion in complex, shear-rate-dependent fluids. We show that using purely planar data significantly underestimates the true value of the shear rate throughout the field. We then use symmetry arguments validated against numerics to show that the 2D measurements can be corrected for out-of-plane effects by applying the incompressibility constraint. Our work shows that fundamental fluid mechanics can be used alongside experimental measurements to improve our understanding of the biomechanics of locomotion.

\section{METHODS}

In order to compare the results of the numerical model directly with our experimental data, we use the same swimming movie to construct experimental velocity fields and to obtain the motion of the nematode, which provides the nematode's geometry and kinematics for the numerical simulations.

Particle tracking procedures around swimmers entail a delicate balance between maximizing the quantity of statistics from which to construct a velocity field and the precision of those measurements. For $C$. elegans, one needs enough statistics to construct a smooth, differentiable velocity field, yet the most precise particle tracking data are located strictly at the nematode $z$ midplane (direction across the depth of field). Additionally, due to the fact that our microscope objectives have a depth of field of approximately $20 \mu \mathrm{m}$, our experiments represent a depth-averaged 2D slice of a 3D flow field over this thickness.

In this paper, simulation provides benchmark flow fields, allowing greater selectivity for our particle identification and tracking algorithms. This selection ensures that we only choose particles close to the $z$ midplane, shifting the balance from maximizing the number of statistics to maximizing the precision of our measurements. In regions where we have limited data, we use gentle maximum value and bilateral filtering algorithms to smooth our experimental results so that they can be differentiated. 


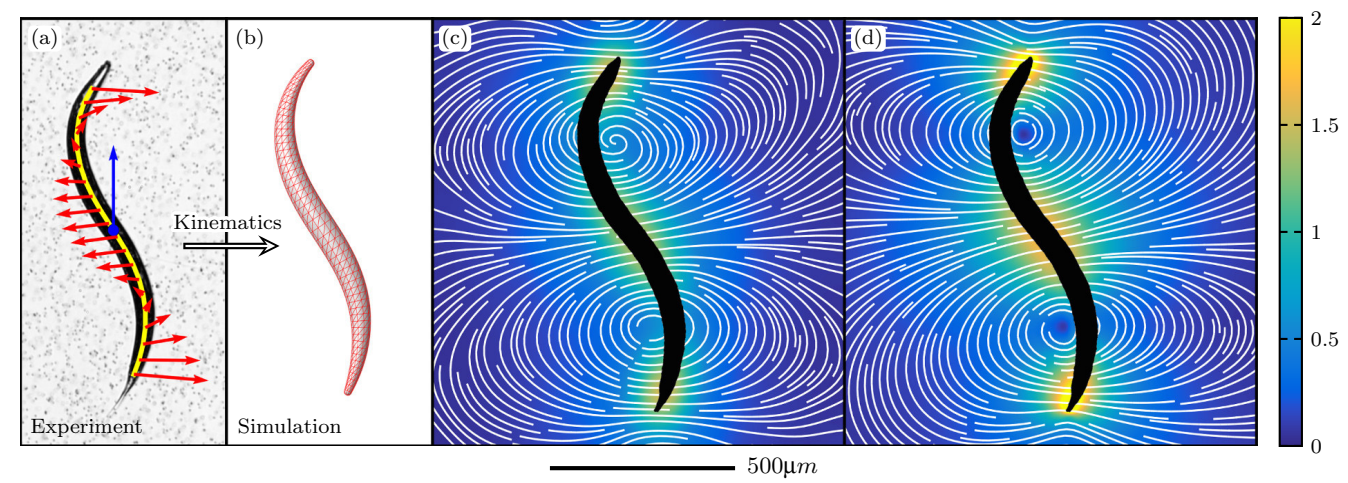

FIG. 2. (a) Image of the nematode C. elegans. The yellow line shows the "skeletonized" body, and the red arrows denote the velocities of each body segment. The blue arrow indicates the swimming direction. (b) Threedimensional mesh constructed from the experimentally obtained body shape and kinematics of the nematode for the same snapshot. (c) Experimentally measured streamlines for one snapshot of swimming C. elegans, produced using particle tracking velocimetry techniques. (d) Flow field produced using the reconstructed 3D mesh and numerical model for the same snapshot. Color bar: speed mm/s.

\section{A. Data acquisition and processing}

Experiments with $C$. elegans are performed in Newtonian, water-like $(\mu \approx 1 \mathrm{mPa} \cdot \mathrm{s}$ and $\rho \approx 10^{3} \mathrm{~kg} / \mathrm{m}^{3}$ ) M9 buffer solutions [7] in a sealed fluid chamber $20 \mathrm{~mm}$ in diameter and $1 \mathrm{~mm}$ in depth. Images are captured using standard bright-field microscopy (Infinity K2/SC microscope with a CF-4 objective and an IO Industries Flare M180 camera at 150 frames per second). The depth of focus of the objective is approximately $20 \mu \mathrm{m}$, and we ensure that the focal plane is in the middle of the chamber in order to minimize wall effects.

The nematode, which is $1 \mathrm{~mm}$ in length and $75 \mu \mathrm{m}$ in diameter, beats primarily in the observation plane; the out-of-plane beating amplitude is less than $6 \%$ of its in-plane motion, and therefore confinement effects are minimal [21]. Given that the flow decay is expected to be exponential, we also anticipate negligible influence of the chamber boundaries on the flow field (see Fig. 4) [22]. All data presented here pertain to nematodes swimming at the center of the fluidic chamber, and out-of-plane recordings are discarded to avoid nematode-wall interactions and to minimize three-dimensional flow effects.

In-house software is used to track the swimming motion of $C$. elegans. The position of the nematode's centroid is differentiated with respect to time to obtain swimming speed $(U \approx 0.3 \mathrm{~mm} / \mathrm{s}$ ), the head position is used to compute an average amplitude $(A \approx 0.25 \mathrm{~mm})$, and a comparison of periodic body shapes is used to estimate the nematode's beating frequency $(f \approx 2 \mathrm{~Hz})$. Beyond average kinematic properties, the nematode body contour is automatically extracted for each image frame, skeletonized [yellow line in Fig. 2(a)], and divided into segments that are tracked to obtain local body velocities. Sample velocity vectors of the body are shown in Fig. 2(a) in red. This body shape and associated velocities are used to create an approximate 3D model of the nematode and its surface velocities, as illustrated in Fig. 2(b).

Particle tracking velocimetry is used to measure the velocity fields generated by swimming C. elegans. In short, we seed the chamber with $3 \mu \mathrm{m}$ polystyrene tracer particles [Fig. 2(a)] that are tracked with in-house algorithms. We track the flow for approximately eight beat cycles, and divide each cycle into approximately 50 phases. Because the nematode's swimming stroke is highly periodic ( $\sim 2 \mathrm{~Hz}$ ) [21], we can construct a "master" swimming cycle using a least-squares fit of the nematode's body shapes. This phase-averaging technique considerably improves the spatial resolution of the experimental velocity fields. In order to estimate the flow field around the nematode's body more accurately, we include velocities within the contour of the swimmer-fluid interface, assuming a 
no-slip boundary condition. Finally, the data points for each phase, including tracking plus boundary conditions, are averaged into gridded spaces of size $13.2 \mu \mathrm{m}$. The resulting experimental streamlines are illustrated in Fig. 2(c) for one particular phase.

\section{B. Numerical model}

In the experimental setup, C. elegans moves through a water-like Newtonian buffer solution [7]. Thus, the characteristic Reynolds number for the flow, $\operatorname{Re}=\rho U L / \mu$, is less than unity $(\operatorname{Re} \leqslant 0.3$ ). As such, the dynamics of the flow driven by the swimming nematode is well-modeled by the Stokes flow equations

$$
\mu \nabla^{2} \mathbf{u}-\nabla p=0, \quad \nabla \cdot \mathbf{u}=0,
$$

where $\mathbf{u}$ is the fluid velocity and $p$ the dynamic pressure.

In order to solve Eqs. (1) in the fluid surrounding the nematode, we employ the regularized stokeslet boundary element method $[23,24]$. The velocity throughout the domain is given by integrals of stokeslets $\mathbf{S}$ and stresslets $\mathbf{T}$ over the nematode's surface, $S$

$$
\lambda u_{j}\left(\mathbf{x}_{0}\right)=\int_{S} S_{i j}^{\epsilon}\left(\mathbf{x}, \mathbf{x}_{0}\right) f_{i}(\mathbf{x})-u_{i}(\mathbf{x}) T_{i j k}^{\epsilon}\left(\mathbf{x}, \mathbf{x}_{0}\right) n_{k}(\mathbf{x}) d S_{x},
$$

for unknown surface tractions, $\mathbf{f}$, and surface velocity $\mathbf{u}$ specified from experimental data.

Note that in the majority of implementations of the method of regularized stokeslets, the stresslet "double layer" $u_{i}(\mathbf{x}) T_{i j k}^{\epsilon}\left(\mathbf{x}, \mathbf{x}_{0}\right) n_{k}(\mathbf{x})$ term in Eq. (2) is eliminated, and the constant $\lambda=1$ [25]. However the surface tractions solved for in the simplified "single layer" formulation are a modified force density dependent upon a fictitious "complementary" flow inside the worm. In order to provide a general method that might in the future be used to examine the energetics of locomotion and force generation inside the worm, the full formulation is used.

In our case, the constant $\lambda$ is given at leading order by $\lambda \approx 1 / 2+\kappa \epsilon / 4$, where $\kappa$ is the mean local curvature of the surface at $\mathbf{x}_{0}$ [26]. We use the regularized form of the stokeslet $S_{i j}^{\epsilon}$ and stresslet $T_{i j k}^{\epsilon}[23]$,

$$
\begin{aligned}
S_{i j}^{\epsilon}\left(\mathbf{x}, \mathbf{x}_{0}\right) & =\frac{\delta_{i j}\left(r^{2}+2 \epsilon^{2}\right)+r_{i} r_{j}}{r_{\epsilon}^{3}}, \\
T_{i j k}^{\epsilon}\left(\mathbf{x}, \mathbf{x}_{0}\right) & =-\frac{6 r_{i} r_{j} r_{k}}{r_{\epsilon}^{5}}-\frac{3 \epsilon^{2}\left(r_{i} \delta_{j k}+r_{j} \delta_{i k}+r_{k} \delta_{i j}\right)}{r_{\epsilon}^{5}},
\end{aligned}
$$

derived from a regularization of the dirac delta function of the form

$$
\phi_{\epsilon}\left(\mathbf{x}-\mathbf{x}_{0}\right)=\frac{15 \epsilon^{4}}{8 \pi r_{\epsilon}^{7}}, \quad r_{\epsilon}^{2}=r^{2}+\epsilon^{2},
$$

where $r_{i}=\left(\mathbf{x}-\mathbf{x}_{0}\right)_{i}, r=\left|\mathbf{x}-\mathbf{x}_{0}\right|$ and $\epsilon \ll 1$ (with $\epsilon=10^{-4} L$ in our simulations). The implementation uses routines adapted from BEMLIB [27] and the authors' boundary element library RegBEM Phoretic [28], which employs a linear panel representation of the unknown surface tractions $f_{i}(\mathbf{x})$ with adaptive Fekete quadrature for near-singular element integrals [26].

The nematode geometry is meshed with piecewise-quadratic triangular elements [Fig. 2(b)] using a custom routine which extrudes a circle of radius $a(s)$ along the experimentally-captured nematode centerline and caps the head and tail of the worm with a section of a sphere. The radius $a(s)$ is given as a function of arc length $s$ by fitting a quadratic through the nematode's radius at the midpoint $a_{1}$ and the head $a_{2}$, so that for a nematode of length $L$ we have

$$
a(s)=\frac{4\left(a_{2}-a_{1}\right)}{L^{2}}\left(s-\frac{L}{2}\right)^{2}+a_{1} .
$$




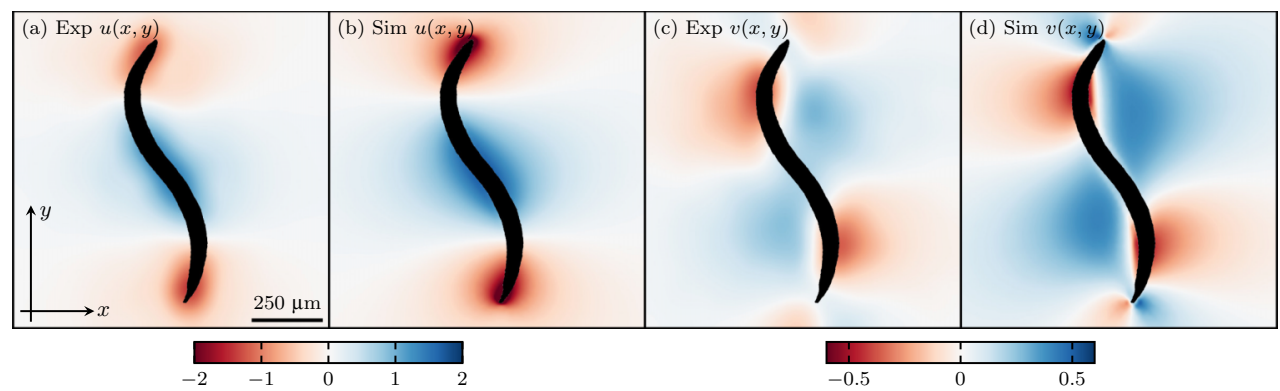

FIG. 3. Spatial distribution of the (a) experimentally-measured $x$-component $u(x, y)$, (b) simulated $x$ component, (c) experimentally-measured $y$-component $v(x, y)$, and (d) simulated $y$-component. Experimental measurements demonstrate good agreement with simulation. Color bars: component velocity magnitude $\mathrm{mm} / \mathrm{s}$.

The velocity boundary condition (no-slip) is then imposed on the mesh surface directly from experimental data via time-centered finite differences of the nematode centerline.

\section{RESULTS AND DISCUSSION}

\section{A. Comparison of experiments and simulations}

Figure 2 shows the streamlines computed experimentally using particle tracking velocimetry (c) and numerically using simulated flow fields (d). These streamlines show strong agreement, with both methods capturing head and tail vortices of similar shape and size. A visual comparison of the $x$ - and $y$-components of the velocity field, $u$ and $v$, respectively, again shows very good agreement between experiment and simulation (Fig. 3). In order to quantify this comparison, Figs. 4(a)-4(c) shows the distributions of velocity components $u$ and $v$ and speed $|\mathbf{u}|$ for experimental and simulated fields. These plots show that the experiments capture the majority of velocities, with the exception of the highest velocities corresponding to points closest to the nematode, where it is difficult to obtain accurate particle tracks. Experiments also predict a larger proportion of zero velocities, which is again associated with the difficulty of extracting smooth, small, but nonzero, velocities from noisy particle data.

Finally, we calculate the spatial decay of the flow speed away from the body of the swimmer. For an undulatory swimmer, we expect to observe an exponential flow decay [22],

$$
\frac{|\mathbf{u}|}{\left|\mathbf{u}_{b}\right|}=\exp \left(-\frac{2 \pi r}{\alpha L}\right)
$$

(a)

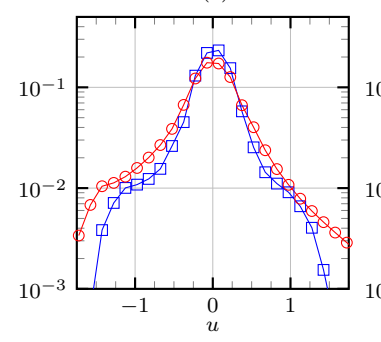

(b)

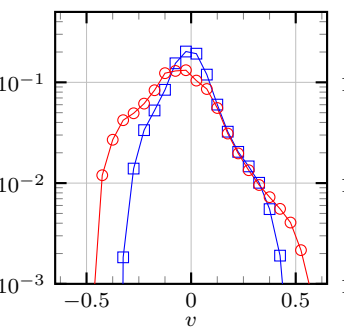

(c)

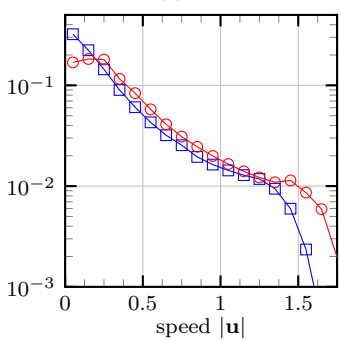

(d)

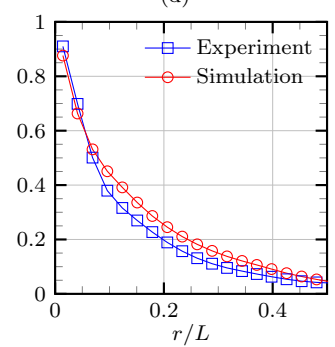

FIG. 4. Comparison of flow structure between experiment and simulation: (a) distribution of $u$, (b) distribution of $v$, (c) distribution of speed $|\mathbf{u}|$, and (d) decay rate $|\mathbf{u}| /\left|\mathbf{u}_{b}\right|$. 
where $|\mathbf{u}|$ is a velocity measurement, $\left|\mathbf{u}_{b}\right|$ is the speed of the swimmer's body, assuming a no-slip boundary condition, $r$ is the normal distance from each velocity measurement to the swimmer's body, $L$ is a characteristic length scale of the swimmer (we take $L \approx 1 \mathrm{~mm}$ ), and $\alpha$ is an exponential parameter that specifies the rate of decay. We find that the simulations and experiments are in good agreement with $\alpha_{\exp }=0.74$ and $\alpha_{\text {sim }}=0.85$, showing a difference of just $13 \%$ in the exponent (Fig. 4, last panel).

\section{B. Shear rate calculation and correction}

A particularly salient characteristic of the flow field is the spatial ditribution of shear rate, $\dot{\gamma}=\sqrt{\varepsilon(\mathbf{u}): \boldsymbol{\varepsilon}(\mathbf{u}) / 2}$ with $\boldsymbol{\varepsilon}(\mathbf{u})=\nabla \mathbf{u}+\nabla \mathbf{u}^{T}$, which is important for calculation of power dissipation and the energetics of locomotion, and is also relevant for studies on locomotion in complex, (shear) rate-dependent fluids. Since the beat-pattern of the nematode is planar, there is no flow in the $z$ direction in the swimmer midplane. However, the $z$ derivatives of the midplane velocity will make, in general, nontrivial contributions to the shear rate. Writing the shear rate in $2 \mathrm{D}$ and $3 \mathrm{D}$ explicitly, we see

$$
\begin{aligned}
& \dot{\gamma}_{2 \mathrm{D}}=\left[2 u_{x}^{2}+\left(u_{y}+v_{x}\right)^{2}+2 v_{y}^{2}\right]^{1 / 2}, \\
& \dot{\gamma}_{3 \mathrm{D}}=\left[2 u_{x}^{2}+\left(u_{y}+v_{x}\right)^{2}+2 v_{y}^{2}+2 w_{z}^{2}+\left(u_{z}+w_{x}\right)^{2}+\left(v_{z}+w_{y}\right)^{2}\right]^{1 / 2},
\end{aligned}
$$

where $u, v, w$ are the $x, y, z$ components of the velocity field, and subscripts denote derivative components.

Because of the additional terms in the 3D formula, we expect that calculation of the shear rate from $2 \mathrm{D}$ particle tracking flow fields will likely result in a systematic underestimate of the true 3D value. Figure 5(a) shows that this is indeed the case; the spatial distribution of the relative percentage error in the simulated shear rate calculated with the 2D formula (7a) compared with the true 3D value spatial distribution of the shear rate as calculated by the $3 \mathrm{D}(7 \mathrm{~b})$ is significant, even reasonably far from the worm, and is around $30 \%$ close to the worm. Thus, we see that we have significant errors throughout the field, but particularly close to the worm where calculations of shear rate are of particular interest.

We wish to correct for this error without resorting to Boundary Element calculations and therefore require estimates of the unknown quantities in the $3 \mathrm{D}$ formula (7b). Since the worm kinematics are planar, we have the symmetry $z \rightarrow-z$, and so there is no $z$ flow in the midplane and the quantities
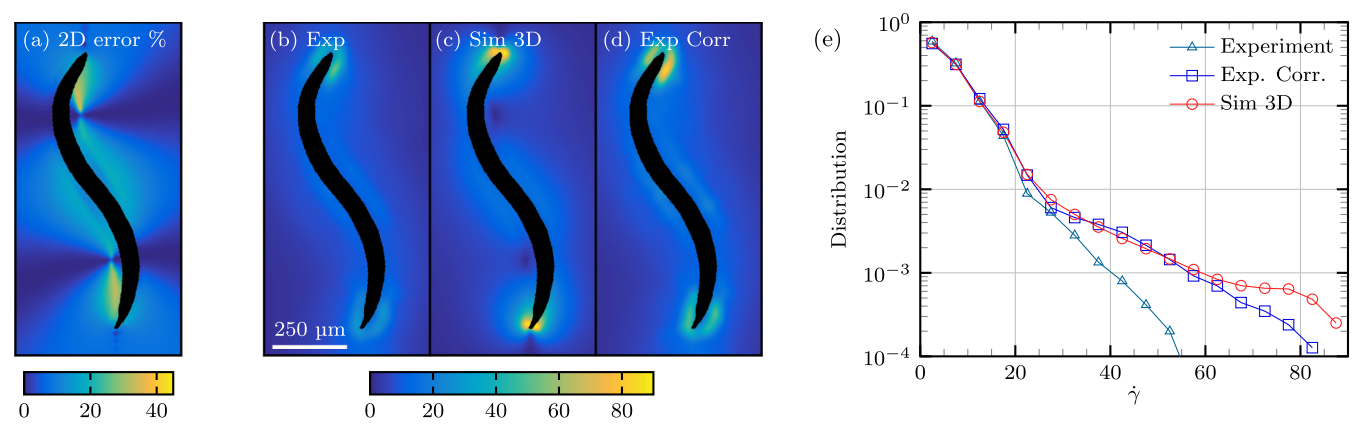

FIG. 5. Shear rate error, calculation, and correction: (a) The percentage relative error in the shear rate as calculated using the 2D formula (7a) for simulated data, showing large errors throughout. (b)-(d) Shear rate field for (b) planar 2D experiments calculated with the 2D formula (7a), (c) 3D simulation calculated with the full formula (7b), and (d) corrected "3D" experiments calculated with the corrected formula (9). (e) Distribution of shear rates for planar experiments, 3D simulation, and corrected experiments. Note the marked improvement in similarity between shear rate distribution for the corrected experiments and that of the full 3D simulation. 
$w_{x}$ and $w_{y}$ are zero. Furthermore, the quantities $u_{z}$ and $v_{z}$ are also zero by this symmetry. These observations are confirmed by our numerical simulation, which calculates the above quantities to be zero within numerical error. However, the $z$-component of the velocity $w$ changes sign through the midplane, and thus its $z$ derivative $w_{z}$ makes a significant contribution to the shear rate. The 3D formula ( $7 \mathrm{~b})$ can thus be simplified,

$$
\dot{\gamma}_{\mathrm{pl}}=\left[2 u_{x}^{2}+\left(u_{y}+v_{x}\right)^{2}+2 v_{y}^{2}+2 w_{z}^{2}\right]^{1 / 2} \text {. }
$$

Since the flow is incompressible, we have $\nabla \cdot \mathbf{u}=0$, so that $w_{z}=-\left(u_{x}+v_{y}\right)$, giving the final formula

$$
\dot{\gamma}_{\mathrm{pl}}=\left[2 u_{x}^{2}+2 v_{y}^{2}+\left(u_{y}+v_{x}\right)^{2}+2\left(u_{x}+v_{y}\right)^{2}\right]^{1 / 2}
$$

purely in terms of components obtainable via planar particle tracking. Applying the adjusted formula (9) to our numerical data, the error is eliminated to within $0.01 \%$ which is attributable to the accuracy of our numerical scheme.

The principal advantage of this approach is that it can be applied in just as simple a manner to incompressible non-Newtonian flows, such as shear-thinning and viscoelastic fluids. Such fluids have complex non-Newtonian constitutive laws and do not readily admit simple three-dimensional simulation.

We now directly compute the 2D (7a) and the estimated 3D (9) shear rate field for our experimental data [Fig. 5(b)]. Figure 5 shows a comparison between the "corrected" 3D shear rate field from experiments and the 3D shear rate field from simulations (c,d). We find the corrected field from experiments and the simulated 3D field have a strikingly similar structure and an RMS error of only $14 \%$, compared to an RMS error of roughly $20 \%$ before the correction was applied. We note, however, that there are discrepancies near the head and tail of the worm, where the simulations suggest a slightly higher shear rate $(\approx 10 \%)$; these regions of high velocity near the swimmer-fluid interface are in locations where we expect particle tracking techniques to pose the greatest challenge.

To quantify the differences between the shear rate fields of the simulations and experiments, as well as demonstrate the effectiveness of our correction factor for 2D data, we show the distribution of shear rate for the raw experimental data, corrected experimental data, and the full 3D simulations [Fig. 5(e)]. We find that a 2D calculation of shear rate using our experimental data underestimates shear rate for values of $\dot{\gamma}>25 \mathrm{~s}^{-1}$ compared to the 3D simulated shear rate field. When the Eq. (9) is applied to the experimental data, we capture the same shear rate distribution as the 3D simulations up to $\dot{\gamma}=60 \mathrm{~s}^{-1}$. For larger shear rates $\left(\dot{\gamma}>60 \mathrm{~s}^{-1}\right)$, we see only minor deviations from the $3 \mathrm{D}$ simulations, suggesting that the corrected shear rate field significantly improves our ability to estimate the shear rate magnitude from planar data.

Since the shear rate is calculated using derivatives of the velocity field, small fluctuations or errors in the velocity field are magnified substantially. Nonetheless, we obtain good agreement between experiment and simulation, with errors being confined to regions of high shear rate; importantly, we find that the application of the analytical correction factor greatly improves our estimate of the maximum shear rate around the swimmer, which is particularly useful for swimming applications with non-Newtonian fluids. These results indicate that the local shear rate near a low Reynolds number swimmer is likely much higher than previously thought, due to contributions in the third dimension that planar particle tracking velocimetry does not directly measure. As a result, non-Newtonian effects as a result of locomotion in complex fluids may be much larger than anticipated. Examples include the role of elastic stretching, measured by the Weissenberg number $\mathrm{Wi}=\lambda_{E} \dot{\gamma}$ where $\lambda_{E}$ is the longest relaxation time of the fluid, and shear-thinning viscosity behavior, measured by the Carreau number $\mathrm{Cr}=\lambda_{\mathrm{Cr}} \dot{\gamma}$ where $\lambda_{\mathrm{Cr}}$ is a time scale that represents the onset of shear-thinning effects; our accounting for the shear rate in the third dimension therefore suggests that a planar experimental measurement may under-represent non-Newtonian effects near the body of the swimmer. 


\section{CONCLUSION}

In this work, we used theoretical and numerical techniques to improve the processing of experimentally obtained particle tracking data, producing smooth velocity fields quantifying the flow around the swimming nematode $C$. elegans in a Newtonian solution. We compared our results with a 3D boundary element model of the nematode, generated directly from experimentally-obtained nematode kinematics, finding good agreement between numerics and experiment.

We argued that when calculating derivative flow quantities, the only nontrivial out-of-plane component is $w_{z}$ : the $z$ derivative of the $z$ flow. This observation was validated by simulation, showing that excluding $w_{z}$ when calculating the flow shear rate results in a significant underestimate. Accounting for the $w_{z}$ component via incompressibility from $2 \mathrm{D}$ data eliminated this error in the numerics, and greatly improved the experimental data.

Our work illustrates how theory may be used to improve experimental measurements in biological fluid mechanics and will be directly applicable to investigations of bio-locomotion in complex fluids. Furthermore, we anticipate that as technology for the acquisition and processing of experimental flow fields continues to improve, these out-of-plane effects will represent a hard barrier to increasing the accuracy of results, making such techniques increasingly important.

\section{ACKNOWLEDGMENTS}

T.D.M.-J. is supported by a Royal Commission for the Exhibition of 1851 Research Fellowship and D.A.G. is supported by an NSF Graduate Fellowship. Funding from the European Union (CIG Grant to E.L.) and NSF-CBET-1437482 (to P.E.A.) is gratefully acknowledged. The authors would like to thank the anonymous referees for their helpful comments on the manuscript.

T.D.M.-J. and D.A.G. contributed equally to this work.

[1] L. A. Johnson, G. R. Welch, and W. Rens, The Beltsville sperm sexing technology: high-speed sperm sorting gives improved sperm output for in vitro fertilization and AI, J. Anim. Sci. 77, 213 (1999).

[2] L. Fauci and R. Dillon, Biofluidmechanics of reproduction, Annu. Rev. Fluid Mech. 38, 371 (2006).

[3] M. A. Bees and O. A. Croze, Mathematics for streamlined biofuel production from unicellular algae, Biofuels 5, 53 (2014).

[4] F. Qiu and B. J. Nelson, Magnetic helical micro- and nanorobots: toward their biomedical applications, Engineering 1, 21 (2015).

[5] E. Lauga and T. Powers, The hydrodynamics of swimming microorganisms, Rep. Prog. Phys. 72, 096601 (2009).

[6] E. A. Gaffney, H. Gadelha, D. J. Smith, J. R. Blake, and J. C. Kirkman-Brown, Mammalian sperm motility: Observation and theory, Annu. Rev. Fluid Mech. 43, 501 (2011).

[7] S. Brenner, The genetics of Caenorhabditis elegans, Genetics 77, 71 (1974).

[8] L. Byerly, R. Cassada, and R. Russell, The life cycle of the nematode Caenorhabditis elegans. I. Wild-type growth and reproduction, Develop. Biol. 51, 23 (1976).

[9] J. Gray and H. W. Lissmann, The locomotion of nematodes, J. Exp. Biol. 41, 135 (1964).

[10] J. Korta, D. Clark, C. Gabel, L. Mahadevan, and A. D. T. Samuel, Mechanosensation and mechanical load modulate the locomotory gait of swimming C. elegans, J. Exp. Biol. 210, 2383 (2007).

[11] M. Backholm, R. D. Schulman, W. S. Ryu, and K. Dalnoki-Veress, Tangling of Tethered Swimmers: Interactions Between Two Nematodes, Phys. Rev. Lett. 113, 138101 (2014).

[12] X. N. Shen and P. E. Arratia, Undulatory Swimming in Viscoelastic Fluids, Phys. Rev. Lett. 106, 208101 (2011).

[13] A. Bilbao, E. Wajnryb, S. A. Vanapalli, and J. Blawzdziewicz, Nematode locomotion in unconfined and confined fluids, Phys. Fluids 25, 081902 (2013). 
[14] D. A. Gagnon, N. C. Keim, and P. E. Arratia, Undulatory swimming in shear-thinning fluids: Experiments with Caenorhabditis elegans, J. Fluid Mech. 758, R3 (2014).

[15] J.-S. Park, D. Kim, J. H. Shin, and D. A. Weitz, Efficient nematode swimming in a shear thinning colloidal suspension, Soft Matter 12 (2016).

[16] J. Sznitman, P. Purohit, P. Krajacic, T. Lamitina, and P. Arratia, Material properties of Caenorhabditis elegans swimming at low Reynolds number, Biophys. J. 98, 617 (2010).

[17] B. Thomases and R. D. Guy, Mechanisms of Elastic Enhancement and Hindrance for Finite-Length Undulatory Swimmers in Viscoelastic Fluids, Phys. Rev. Lett. 113, 098102 (2014).

[18] D. Gagnon, X. Shen, and P. Arratia, Undulatory swimming in fluids with polymer networks, Europhys. Lett. 104, 14004 (2013).

[19] T. Majmudar, E. E. Keaveny, J. Zhang, and M. J. Shelley, Experiments and theory of undulatory locomotion in a simple structured medium, J. R. Soc. Interface 9, 1809 (2012).

[20] J. Z. Yuan, D. M. Raizen, and H. H. Bau, High-throughput, motility-based sorter for microswimmers such as C. elegans, Proc. Natl. Acad. Sci. USA 112, 3158 (2015).

[21] J. Sznitman, X.-N. Shen, R. Sznitman, and P. Arratia, Propulsive force measurements and flow behavior of undulatory swimmers at low Reynolds number, Phys. Fluids 22, 121901 (2010).

[22] J. Lighthill, Flagellar hydrodynamics, SIAM Rev. 18, 161 (1976).

[23] R. Cortez, L. Fauci, and A. Medovikov, The method of regularized Stokeslets in three dimensions: Analysis, validation, and application to helical swimming, Phys. Fluids 17, 031504 (2005).

[24] D. J. Smith, A boundary element regularized Stokeslet method applied to cilia- and flagella-driven flow, Proc. R. Soc. Lond. A 465, 3605 (2009).

[25] C. Pozrikidis, Boundary Integral and Singularity Methods for Linearized Viscous Flow (Cambridge University Press, Cambridge, 1992).

[26] T. D. Montenegro-Johnson, S. Michelin, and E. Lauga, A regularised singularity approach to phoretic problems, Eur. Phys. J. E 38, 139 (2015).

[27] C. Pozrikidis, A Practical Guide to Boundary Element Methods with the Software Library BEMLIB (CRC Press, Boca Raton, FL, 2002).

[28] http://uk.mathworks.com/matlabcentral/profile/authors/5102158-thomas-montenegro-johnson. 